\title{
Planejamento Pré-operatório Utilizando Reconstruções 3D e Endoscopia Virtual para a Localização do Seio Frontal
}

\section{Preoperative Planning Using 3D Reconstructions and Virtual Endoscopy for Location of the Frontal Sinus}

\author{
Marcos Jullian Barreto Martins*, Carolina Veras Aguiar*, João Flávio Nogueira Júnior**, \\ João Paulo Saraiva Abreu ***, Moisés Ximenes Feijão***, Isabelle Oliveira Jataí $* * * *$, \\ Arthur Chaves Gomes Bastos****.
}

\author{
* Graduação em Medicina. Residente de 3a. Ano de Otorrinolaringologia do Hospital Geral de Fortaleza - HGF. \\ ** Título de Especialista em Otorrinolaringologia pela ABORL-CCF. Diretor Sinus Centro Médico Otorrinolaringologista do Hospital Geral de Fortaleza - HGF. \\ *** Graduação em Medicina. Residente de 2a. Ano de Otorrinolaringologia do Hospital Geral de Fortaleza - HGF. \\ ***** Graduação em Medicina. Residente de 1a. Ano de Otorrinolaringologia do Hospital Geral de Fortaleza - HGF. \\ Instituição: Hospital Geral de Fortaleza - HGF \& Sinus Centro. \\ Fortaleza / CE - Brasil. \\ Endereço para correspondênica: João Flávio Nogueira - Avenida Pontes Vieira, 2531 - Bairro: São João do Tauape - Fortaleza / CE - Brasil - Telefone: (+55 85) \\ 8152-2322 - http://www.sinuscentro.com.br - E-mail: joaoflavioce@ hotmail.com \\ Artigo recebido em $1^{\circ}$ de Setembro de 2010. Artigo aprovado em 7 de Outubro de 2010.
}

\section{RESUMO}

Introdução:

Reconstruções tridimensionais (3D) geradas de tomografias computadorizadas (TCs) permitem a observação de cavidades e estruturas anatômicas de nosso corpo com riqueza de detalhes. Em nossa especialidade já houve tentativas da realização de endoscopias e laringoscopias virtuais. Entretanto, estas aplicações foram praticamente abandonadas devido à complexidade e necessidade de computadores com alto poder de processamento gráfico.

Objetivo: Demonstrar a confecção de reconstruções 3D a partir de TCs de pacientes em computador pessoal, com programa específico gratuito e compará-las às imagens endoscópicas reais da cirurgia.

Método:

Estudo prospectivo em que os arquivos próprios das TCs de 10 pacientes foram reconstruídos com programa Intage Realia, versão 2009, 0, 0, 702 (KGT Inc, Japão). As reconstruções foram realizadas antes das cirurgias e uma endoscopia virtual foi feita para se avaliar a região do recesso e seio frontal. Após este estudo, a cirurgia foi realizada e armazenada digitalmente. As imagens endoscópicas reais da região do recesso e seio frontal foram comparadas às imagens virtuais.

Resultados: A reconstrução 3D e endoscopia virtual foram realizadas nos 10 pacientes submetidos à cirurgia. As imagens virtuais apresentavam grande semelhança visual com as imagens das cirurgias reais.

Conclusão:

Demonstramos a possibilidade, com ferramentas relativamente simples e computador pessoal, de se gerar reconstruções 3D e endoscopias virtuais. O conhecimento pré-operatório da localização do caminho de drenagem natural do seio frontal pode vir a gerar benefícios durante a realização das cirurgias. Entretanto, mais estudos devem ser desenvolvidos para a avaliação do real papel destas reconstruções 3D e endoscopias virtuais.

Palavras-chave: cirurgia assistida por computador, cirurgia vídeo-assistida, tomografia, imagem tridimensional, endoscopia.

\section{SUMMARY}

Introduction:

Computed tomography (TC) generated tridimensional (3D) reconstructions allow the observation of cavities and anatomic structures of our body with detail. In our specialty there have been attempts to carry out virtual endoscopies and laryngoscopies. However, such application has been practically abandoned due to its complexity and need for computers with high power of graphic processing.

Objective: To demonstrate the production of $3 \mathrm{D}$ reconstructions from CTs of patients in personal computers, with a free specific program and compare them to the surgery actual endoscopic images.

Method: Prospective study in which the CTs proper files of 10 patients were reconstructed with the program Intage Realia, version 2009, 0, 0, 702 (KGT Inc., Japan). The reconstructions were carried out before the surgeries and a virtual endoscopy was made to assess the recess and frontal sinus region. After this study, the surgery was digitally performed and stored. The actual endoscopic images of the recess and frontal sinus region were compared to the virtual images.

Results: The 3D reconstruction and virtual endoscopy were made in 10 patients submitted to the surgery. The virtual images had a large resemblance with the actual surgical images.

Conclusion: With relatively simple tools and personal computer, we demonstrated the possibility to generate $3 \mathrm{D}$ reconstructions and virtual endoscopies. The preoperative knowledge of the frontal sinus natural draining path location may generate benefits during the performance of surgeries. However, more studies must be developed for the evaluation of the real roles of such 3D reconstructions and virtual endoscopies.

Keywords: computer assisted surgery, video-assisted surgery, tomography, tridimensional image, endoscopy. 


\section{INTRODUÇÃO}

O uso e as aplicações das reconstruções tridimensionais (3D) geradas a partir de arquivos próprios (DICOMS) de tomografias computadorizadas (TC) ou ressonâncias magnéticas (RM) está se expandindo recentemente. Estas reconstruções permitem aos médicos a observação de cavidades e estruturas anatômicas de nosso corpo com incrível riqueza de detalhes, sendo capazes de exibir até mesmo as texturas de diferentes tecidos $(1,2)$.

As aplicações para estas reconstruções têm variado de simples ilustrações em exames até para o auxílio no diagnostico e planejamento pré-operatório nas mais diversas especialidades médicas.

Alguns exemplos podem ser encontrados nas colonoscopias virtuais, realizadas por alguns centros de imagem em nosso país e nas TCs de alta resolução para a avaliação das artérias coronárias (3).

Em nossa especialidade já houve algumas tentativas da realização de endoscopias e laringoscopias virtuais. Entretanto, estas aplicações foram praticamente abandonadas devido à complexidade e necessidade de computadores com alto poder de processamento gráfico (4).

Entretanto, com a evolução dos computadores e programas próprios para leitura e reconstrução dos arquivos DICOMS, atualmente alguns autores têm resgatado o uso destas reconstruções 3D para a avaliação e planejamento pré-operatório para especialmente as cirurgias otológicas e do recesso e seio frontal $(5,6)$.

Estas reconstruções 3D podem oferecer informação potencialmente mais útil do que as obtidas com as visões 2D nos planos axial, coronal e sagital. Estas imagens obtidas nos três diferentes planos: axial, coronal e sagital são úteis na análise da anatomia do recesso e seio frontal. Entretanto estas imagens bidimensionais são de uma compreensão mais difícil para os cirurgiões (7). BAKKER et al. Realizaram uma pesquisa com cirurgiões sobre o que seria mais difícil para o aprendizado e realização de cirurgias endoscópicas nasosinusais seguras (8). O que foi considerado de mais difícil aprendizado pelos cirurgiões pesquisados foi: "a orientação espacial, ou seja, fazer uma representação mental 3D a partir de uma tomografia computadorizada 2D". E o que foi considerado como o segundo mais difícil foi: "entender a relação entre os planos da tomografia computadorizada com as imagens reais endoscópicas" (8).

Vivemos em um mundo tridimensional e apesar de grande parte dos endoscópios ainda não permitirem a realização de cirurgias estereoscópicas 3D, as imagens obtidas nas cirurgias endoscópicas tradicionais nos permitem a criação de relações 3D em nossa mente. Estas relações podem ajudar na realização de tarefas particulares e a excelente preparação pré-operatória pode algumas vezes evitar complicações muitas vezes desafiadoras (9).

Temos estudado a utilidade das ferramentas de reconstrução 3D para as cirurgias otológicas e do recesso e seio frontal. Na literatura encontramos desde o planejamento pré-operatório até outras aplicações tais como estadiamento de doenças, medição pré-operatória para confecção de próteses, análises do fluxo aéreo e o próprio ensino a médicos residentes $(5,6,8,10)$.

A avaliação pré-operatória para as cirurgias do seio frontal através de reconstruções 3D a partir de arquivos próprios de TC (DICOMS) pode permitir ao cirurgião adquirir mais informações do que com as tradicionais imagens bidimensionais obtidas com os três diferentes planos: axial, coronal e sagital.

Apesar de ser possível a visualização de todo o caminho de drenagem do seio frontal e de sua orientação e planejamento pré-operatório através das tradicionais TC bidimensionais, elegantemente já descrita por vários autores, dentre eles Wormald et al $(9,11)$, o uso de ferramentas 3D pode eliminar o elemento de incerteza no achado correto do caminho de drenagem natural do seio frontal.

Utilizando estas ferramentas 3D é possível a visualização dos vários orifícios presentes na região do próprio recesso, seguindo-os para a análise de qual é o orifício correto para a drenagem do seio frontal.

O objetivo deste trabalho consiste em:

a) Demonstrar a confecção destas reconstruções $3 \mathrm{D}$ a partir de TCs de pacientes submetidos a cirurgias endoscópicas nasosinusais da região do recesso e seio frontal realizadas em computador pessoal, com programa específico.

b) Comparar estas imagens virtuais às imagens endoscópicas reais obtidas durante a cirurgia.

\section{MÉTOdO}

Após liberação pelo comitê de ética local, foram obtidos arquivos DICOM dos 10 pacientes arregimentados para o estudo. O critério de inclusão foi o de pacientes que iriam se submeterà cirurgia endoscópica dos seios paranasais que também incluísse o seio frontal. O critério de exclusão foi o de pacientes que não seriam submetidos à cirurgia endoscópica do seio frontal. 
As pastas contendo os arquivos foram salvas no computador pessoal e variavam de tamanho entre 150 e $250 \mathrm{Mb}$. As TCs foram solicitadas aos pacientes préoperatoriamente e foram realizadas em diferentes equipamentos "multi-slice". Os exames apresentavam cortes que variavam entre 0,5 e 1,3 $\mathrm{mm}$ de espessura.

Os arquivos próprios (DICOMS) das referentes TCs foram reconstruídos com programa de computador Intage Realia, versão 2009, 0, 0, 702 (KGT Inc, Japão), utilizandose computador pessoal com sistema operacional Microsoft Windows 7 (Figura 1).

Utilizamos as seguintes ferramentas. O programa está na língua inglesa, entretanto traduzimos as ferramentas para o Português para melhor compreensão.

1) Reconstrução multiplanar (multiplanar reconstruction): os arquivos DICOM originais das tomografias computadorizadas são em plano axial. Utilizando esta ferramenta, criamos as reconstruções coronais e sagitais, com imagens bem definidas (Figura 2). A isto chamamos de reconstrução triplanar ou multiplanar, que são comumente utilizadas nas estações de trabalho de radiologistas ou em navegadores cirúrgicos para diagnóstico e orientação cirúrgica. Entretanto, estas reconstruções multiplanares não ofereciam visualização3D e já são popularmente utilizadas para o planejamento pré-operatório de pacientes.

2) Controle de contraste, brilho, sombras $e$ segmentação: o "shaded surface displays" é uma ferramenta que controla o brilho, contraste e sombras das imagens geradas a partir dos arquivos DICOM. A alteração destes parâmetros possibilita a criação de imagens com diferentes janelas, o que pode facilitar a identificação de tecidos ósseos e partes moles. O trabalho foi realizado a partir das densidades dos tecidos e nos permitiu marcar e colorir manualmente ou automaticamente (em áreas principalmente de densidade aérea) regiões com densidades ósseas, aéreas e de partes moles (doenças). Esta ferramenta de segmentação também foi importante para suprimir estas áreas de doença para que pudéssemos ter visualização adequada da região estudada.

3) Renderização de volume (Volume rendering): as imagens geradas a partir de arquivos DICOM foram transformadas em volume 3D (Figura 3) que poderia ser rotacionado em qualquer plano e "dissecado" virtualmente utilizando-se ferramenta de corte, que poderia também ser em qualquer plano, direção ou angulação, dependendo apenas de ajustes realizados pelo operador do programa de computador. Utilizamos com frequência esta ferramenta. O volume criado a partir dos arquivos DICOM foi rotacionado para o ângulo que fosse compatível com a apresentação do paciente à cirurgia. A partir deste plano,

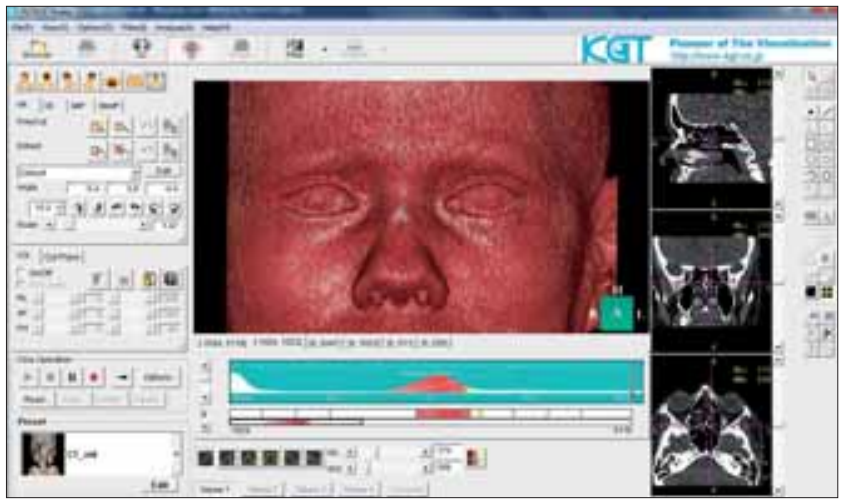

Figura 1. Imagem da tela dos comandos do software utilizado.

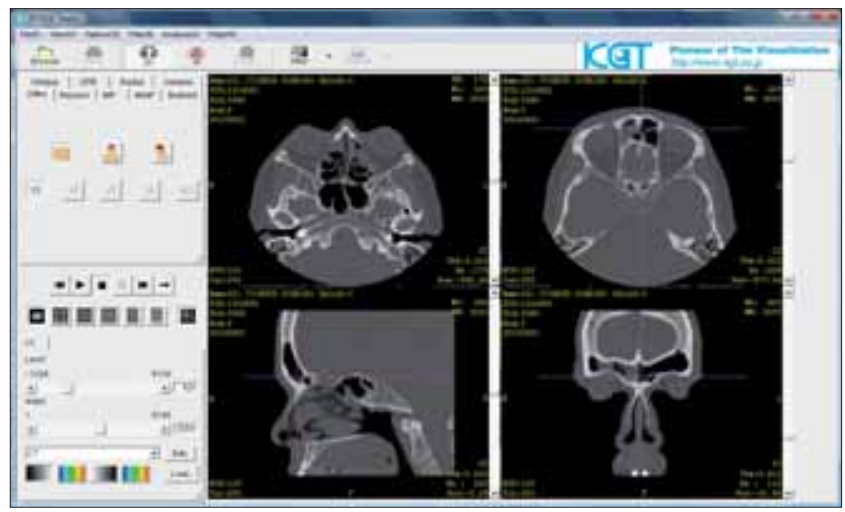

Figura 2. Imagem capturada da tela do computador com programa mostrando reconstruções coronais e sagitais, ou seja, as imagens nos três planos (triplanares), porém em 2D.

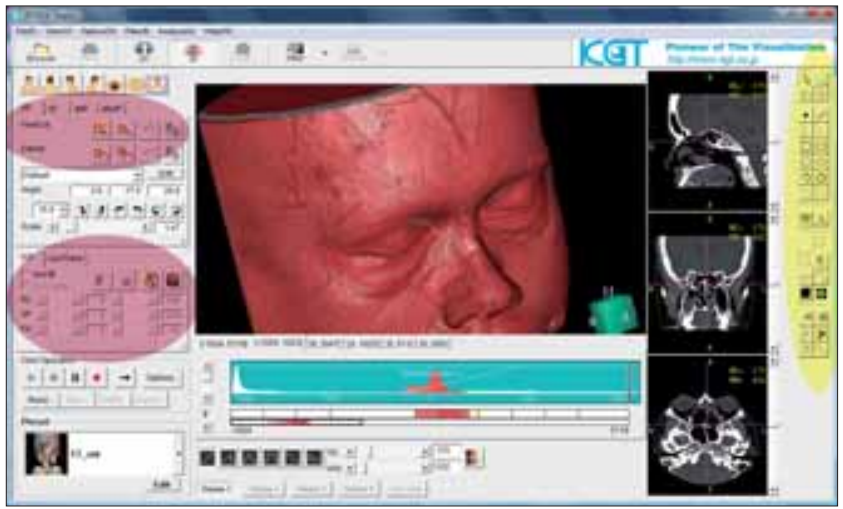

Figura 3. Imagem da tela do computador com programa mostrando renderização de volume 3D. Nas elipses roxas se visualizam os comandos para "dissecção" virtual, cortes livres ("free cuts"), além de controles para rotação e orientação deste volume 3D. Note elipse amarela com comandos para seleção (que pode ser manual ou automática) de áreas tanto nas reconstruções $2 \mathrm{D}$, quanto nas reconstruções de volume 3D, para posterior segmentação e/ou supressão de algumas densidades. 
dissecções eram realizadas pelo volume tanto no sentido superior, quanto inferior, já com as densidades das áreas ósseas, aéreas e partes moles marcadas com diferentes cores. Isto nos possibilitou uma visualização $3 \mathrm{D}$ da região do recesso do seio frontal, permitindo um estudo detalhado dos possíveis canais de drenagem do seio frontal.

4) "Fly through": esta ferramenta nos permitiu uma movimentação dentro do volume criado, simulando uma imagem endoscópica ou uma endoscopia virtual (Figura 4). A partir de comandos no programa de computador selecionando a opção VE (virtual endoscopy) obtemos uma visualização endoscópica das configurações dos óstios do recesso frontal. Em pacientes com menos doença no seio frontal (edema de mucosa, dentre outras) foi mais fácil e conseguimos realizar esta tarefa em menos tempo, pois não precisamos segmentar e/ou suprimir áreas de doença.

Fazendo a endoscopia virtual foi importante manter uma orientação que deveria ser a mesma daquela utilizada nas cirurgias endoscópicas ou endoscopias reais.

As ferramentas disponíveis neste programa permitiam também a movimentação da câmera virtual e mudanças em sua angulação, para que pudéssemos passar por espaços pequenos, o que não seria possível com endoscópios reais.

O ponto de início para a endoscopia virtual ("fly through") foi à região do meato médio, entre o processo uncinado, bolha etmoidal e corneto médio. Após este posicionamento inicial, o campo de visão era movido superiormente no sentido do recesso do seio frontal. À medida que íamos avançando, vários orifícios aparentes apareciam. Mapeamos esta configuração de óstios e capturamos a tela para compararmos à visão endoscópica obtida em cirurgias reais.

Após este estudo da reconstrução 3D, a cirurgia foi realizada e armazenada digitalmente. As imagens endoscópicas reais da região do recesso e seio frontal foram comparadas com as imagens geradas a partir do computador pessoal para avaliar a semelhança.

\section{RESULTADOS}

A reconstrução 3D e a endoscopia virtual foram realizadas em todos os 10 pacientes que posteriormente foram submetidos à cirurgia endoscópica nasossinusal do recesso e seio frontal.

As imagens geradas no computador apresentavam grande semelhança visual com as imagens encontradas nas cirurgias reais (Figuras 5, 6 e 7).

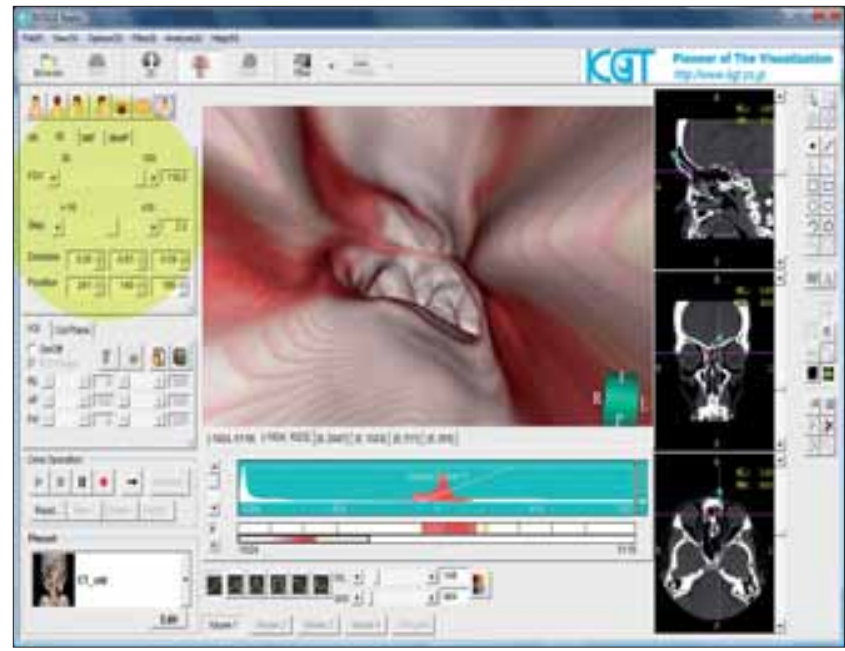

Figura 4. Imagem mostrando exemplo de endoscopia virtual, no comando VE. Note possibilidade de navegação ("fly through") e diferentes angulações da imagem (que pode variar de 30 até 120 graus).

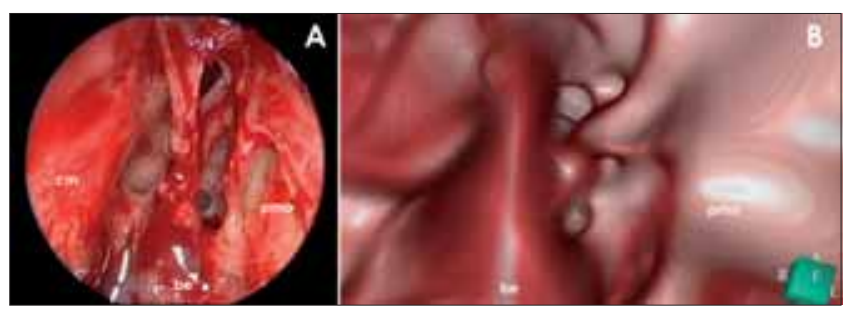

Figura 5. Comparação de imagem de endoscopia real com endoscopia virtual. A: endoscopia real (instrumento de 30 graus) da região do recesso do seio frontal esquerdo. $\mathrm{Cm}$ : corneto médio; be: bolha etmoidal; pmo: parede medial da órbita. B: endoscopia virtual da mesma região. Note semelhança das imagens.

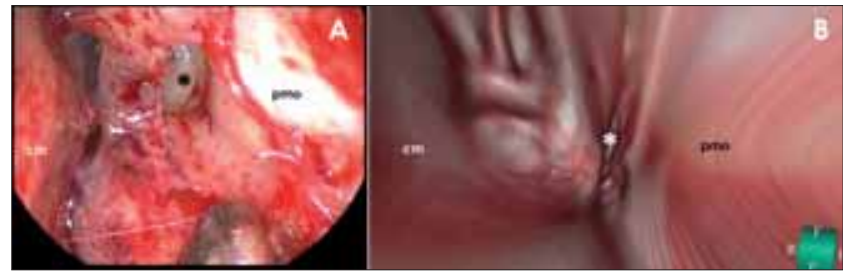

Figura 6. Comparação de imagem de endoscopia real com endoscopia virtual. A: endoscopia real (instrumento de 30 graus) da região do recesso do seio frontal esquerdo. $\mathrm{Cm}$ : corneto médio; pmo: parede medial da órbita. B: endoscopia virtual da mesma região. Note semelhança das imagens.

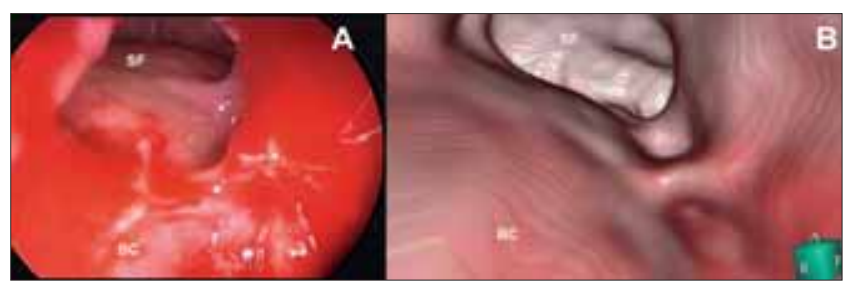

Figura 7. Comparação de imagem de endoscopia real com endoscopia virtual. A: endoscopia real (instrumento de 45 graus) da região do seio frontal (SF) esquerdo da paciente após a abertura. BC: base do crânio na região do ângulo fronto-etmoidal. B: endoscopia virtual da mesma região. Note semelhança das imagens. 
Estas reconstruções foram realizadas sem maiores dificuldades técnicas em computador pessoal, utilizandose programa próprio para leitura e edição de arquivos DICOM.

\section{DISCUSSÃO}

O padrão dos orifícios presentes na área do recesso frontal é muito variável e a localização exata da drenagem natural deste seio não é facilmente determinada pelos tradicionais cortes anatômicos bidimensionais encontrados em uma TC (axial, coronal e sagital) $(7,9,11,12$ ).

Em casos de doença extensa isto se torna mais difícil ainda. Com a reconstrução 3D a partir dos arquivos próprios da TC dos pacientes conseguimos realizar um "mapa" virtual da região do recesso do seio frontal, avaliando a configuração dos óstios e o caminho correto para a drenagem natural do seio frontal. Entretanto, nestes casos em que encontramos doenças muito extensas na região do seio e recesso frontal, a identificação do correto caminho de drenagem do seio frontal foi difícil, mesmo com as reconstruções 3D, visto que para a realização das endoscopias virtuais é necessário alguma presença de ar nas cavidades para uma melhor orientação e navegação. Nestes casos, fomos guiados diretamente pela parte óssea ou utilizamos ferramentas especiais para renderização e supressão da área destas doenças.

Para a correta localização da região do recesso do seio frontal começamos tentando identificar o Agger Nasi, que estava presente em todos os casos estudados e reconstruídos. Esta é tipicamente a primeira célula etmoidal vista no plano coronal no sentido ântero-posterior e é tipicamente confluente com o processo uncinado (12).

O segundo passo foi selecionar o ponto de vista endoscópico do software com a orientação correta, ou seja, a mesma utilizada nas cirurgias tradicionais, para a área entre o processo uncinado, a bolha etmoidal e o corneto médio. A partir deste ponto, movemos o campo de visão no sentido superior para o seio frontal e mapeamos a configuração dos óstios, para que pudéssemos saber em qual orifício se encontrava a drenagem natural do seio frontal.

Anteriormente havíamos tentado a localização do caminho de drenagem natural do seio frontal através dos cortes comuns (2D) de tomografias computadorizadas. Entretanto, mesmo tentando relacionar imagens em planos axial, coronal e sagital, encontramos alguma dificuldade na localização exata do caminho de drenagem natural do seio frontal.

Vale ressaltar que estas reconstruções 3D a partir de arquivos DICOMS próprios das TCs dos pacientes também podem ser realizadas em outros computadores pessoais com outro sistema operacional, como o Macintosh ${ }^{\mathrm{TM}}$, da Apple ${ }^{\mathrm{TM}}$, em programa próprio denominado OsiriX ${ }^{\mathrm{TM}}$, também sendo possível à realização de otoscopias virtuais e planejamento pré-operatório para cirurgias de laringe e de ouvido.

\section{CONCLUSÃO}

Demonstramos a possibilidade, com ferramentas relativamente simples e computador pessoal, de se gerar reconstruções 3D e endoscopias virtuais a partir de arquivos DICOM de TC de pacientes. Nestes pacientes submetidos à cirurgia com o estudo da reconstrução 3D previamente, o seio frontal foi acessado em todos os casos. Entretanto, vale ressaltar, que não realizamos estudos comparativos com grupo de pacientes em que não foi realizada reconstrução $3 \mathrm{D}$.

O conhecimento pré-operatório da localização do caminho de drenagem natural do seio frontal pode vir a gerar benefícios significativos durante a realização das cirurgias tais como menor tempo cirúrgico e maior segurança aos pacientes. Entretanto vale ressaltar que mais estudos devem ser desenvolvidos para a avaliação do real papel destas reconstruções 3D e endoscopias virtuais.

\section{REFERÊNCIAS BIBLIOGRÁFICAS}

1. Anand SM, Frenkiel S, Le BQ, et al. Virtual endoscopy: our next major investigative modality? J Otolaryngol Head Neck Surg. 2009, 38(6):642-5.

2. Wood BJ, Razavi P. Virtual endoscopy: a promising new technology. Am Fam Physician. 2002, 66(1):107-12.

3. McSweeney SE, ODonoghue PM, Jhaveri K. Current and emerging techniques in gastrointestinal imaging. J Postgrad Med. 2010, 56(2):109-16.

4. Gilani S, Norbash AM, Ringl H, et al. Virtual endoscopy of the paranasal sinuses using perspective volume rendered helical sinus computed tomography. Laryngoscope. 1997, 107(1):25-9.

5. Schulze F, Bühler K, Neubauer A, et al. Intra-operative virtual endoscopy for image guided endonasal transsphenoidal pituitary surgery. Int J Comput Assist Radiol Surg. 2010, 5(2):143-54.

6. Lee WT, Kuhn FA, Citardi MJ. 3D computed tomographic analysis of frontal recess anatomy in patients without frontal sinusitis. Otolaryngol Head Neck Surg. 2004, 131(3): 164-73. 
7. Pletcher SD, Hoxworth JM, Goldberg AN, et al. Computed tomography imaging of the paranasal sinuses: direct versus reformatted coronal images. Otolaryngol Head Neck Surg. 2008, 138(6):710-4.

8. Bakker NH, Fokkens WJ, Grimbergen CA. Investigation of training needs for functional endoscopic sinus surgery (FESS). Rhinology. 2005, 43(2):104-8.

9. WormaldPJ. Three-dimensional building block approach tounderstanding the anatomy of the frontal recess and frontal sinus. Operative techniques in Otolaryngology. 2006, 17 : $2-5$.
10. Krüeger A, Kubisch C, Straub G, et al. Sinus endoscopyapplication of advanced GPU volume rendering for virtual endoscopy. IEEE Trans Vis Comput Graph. 2008, 14(6):1491-8.

11. Wormald PJ. Surgery of the frontal recess and frontal sinus. Rhinology. 2005, 43(2):82-5.

12. Park SS, Yoon BN, Cho KS, et al. Pneumatization Pattern of the Frontal Recess: Relationship of the Anterior-toPosterior Length of Frontal Isthmus and/or Frontal Recess with the Volume of Agger Nasi Cell. Clin Exp Otorhinolaryngol. 2010, 3(2):76-83. 